\title{
Effect of Low Temperature Dialysis on The Functional Activity of Complement Activation Pathways during Hemodialysis Procedure \\ Mohammed Nabil Raafat Farhat ${ }^{1}$, Mohammed Elsaed Alshorbagy ${ }^{2}$, Ahmed Mohamed abd-Elfattah Al Ashkar ${ }^{1}$, Mahmoud El-Sayed Lotfy Hassan ${ }^{1}$ \\ Departments of ${ }^{1}$ Internal Medicine and ${ }^{2}$ Clinical Pathology \\ Faculty of Medicine, Al-Azhar University, Cairo, Egypt \\ *Corresponding author: Mahmoud El-Sayed Lotfy Hassan, Mobile: +201097870479, E-Mail: dr.lot64@gmail.com
}

\begin{abstract}
Background: low dialysate temperature dialysis has been used successfully in treatment of intradyalytic hypotension and resistant pruritus. In both conditions complement activation was proposed as one pathological mechanisms for their development. to our knowledge, no study has been done to assess the effect of low dialysate temperature on the individual complement activation pathway. Objective: to assess the effect of low dialysate temperature on complement activation pathways. Patients and Methods: this case control study included 20 patients, on regular hemodialysis at Alhusein University Hospital.

Results: the mean age of our patients was $48.8 \pm 10$ years, 11 males and 9 females, their mean BMI was $22 \pm 4 \mathrm{~kg} / \mathrm{m}^{2}$, their mean blood pressure 120/80 $\pm 20 / 20$, all patients received hemodialysis via arterio-venous fistula. The main cause of hemodialysis is chronic renal failure due to hypertension in 8 patients, glomerulonephritis in 4 patients, polycystic kidney in 2 patients, hyperurecemic nephropathy in 2 patients, analgesic abuser in 1 patient, and chronic pyelonephritis in 1 patient.
\end{abstract}

Conclusion: cold hemodialysis is associated with activation of complement system.

Keywords: hemodialysis, lactin pathway.

\section{INTRODUCTION}

under normal physiological conditions, complement activation leads to a proteolytic cascade resulting in immune cell activation, rapid opsonization and the elimination of microorganisms. however, excessive complement activation, especially in the generation of the c3a, c5a and c5b-complexes, are lifethreatening for patients on hemodialysis(HD) ${ }^{(\mathbf{1})}$.

the c3a, c5a and c5b-9 complexes are generated from the activation of $\mathrm{c} 3$ and $\mathrm{c} 5$ via three complement activation pathways, the classical pathway (cp), alternative pathway (Ap) and lactin pathway $(\mathrm{LP})^{(1)}$.

all over the last ten years complement activation have been linked to various clinical outcomes in hemodialysis patients such as malnutrition, muscle wasting, oxidative stress, endothelial and immune dysfunction, leukopenia, anaphylactoid reactions, amyloidosis, hypo/hypertension, headache, fever, sleep disturbances, and increase risk for atherosclerosis and myocardial infarction ${ }^{(2,3)}$.

Despite reduced induction of complement activity by newer modified cellulose or synthetic membranes, hemodialysis patients are still consistently exposed to low-level complement activity during their frequent treatment sessions, which likely contributes to their chronically acute inflammatory state as described above. As complement acts upstream of many inflammatory pathways, modalities designed to reduce complement activity, and in turn beneficially regulate related cytokine and coagulation networks, would be expected to improve the overall condition of hemodialysis patients and reduce inflammation-related complications ${ }^{(4)}$. low dialysate temperature dialysis has been used successfully in treatment of intradyalytic hypotension and resistant pruritus. In both conditions complement activation was proposed as one pathological mechanisms for their development ${ }^{(5)}$.

to our knowledge, no study has been done to assess the effect of low dialysate temperature on the individual complement activation pathway.

\section{AIM OF THE WORK}

It is to assess the effect of low dialysate temperature on complement activation pathways.

\section{PATIENTS AND METHODS}

This case control study included 20 patients, on regular hemodialysis at Alhusein University Hospital, from $1 / 7$ to $5 / 72018$.

Inclusion criteria: being on dialysis for more than 6 months, cold hemodialysis was prescribed to prevent intradialytic hypotension or alleviate uremic pruritus.

Exclusion criteria: being age less than 18 years or more than 60 , patients with active sepsis, patients with malignancy, organ failure rather than renal failure, diabetes, dialysis via catheter, fistula malfunction.

Institutional Ethics approval: the Ethical Committee of the Alhussein University Hospital had approved this study. An informed consent was taken from the patients.

\section{All the subjects underwent the following:}

in all selected patients the followings were done on admission:

. demographic data: age and sex. cause of chronic renal failure: hypertension, glomerulonephritis, analgesic abuser, chronic pyelonephritis, and obstructive uropathy. past history of: acute renal failure, autoimmune disease, diabetes mellitus. vital signs: heart rate, respiratory rate, 
blood pressure, and temperature. local examination: skin: AV fistula, skin scratch of itching.

\section{Laboratory investigations:}

(1) C3 measures: microtiter plates (Immunoplate Maxisorp; Nunc, Wiesbaden, Germany) were coated overnight with $100 \mathrm{~d}$ of antibody 13/15 at $5 \mathrm{j} . \mathrm{tg} / \mathrm{ml}$ in $0.05 \mathrm{M}$ carbonate buffer $\mathrm{pH}$ 10.6. After washing in phosphate buffered saline (PBS, $\mathrm{pH}$ 7.2) non-specific binding capacity was blocked with $0.05 \mathrm{M}$ carbonate buffer $\mathrm{pH} 10.6$ containing 1\% (wt/vol) gelatin. After washing with PBS/0.05\% Tween-20 100 p.l samples in duplicate 1:50 dilutions in PBS/0.05\% Tween-20/0.02 M EDTA were added for two hours at room temperature. After washing with PBSiTween-20 $100 \mathrm{jd}$ of peroxidase- conjugated rabbit anti-human C3d (Dakopatts) in PBS/ Tween-20 were added and incubated for another one hour at room temperature. Finally, streptavidin-horserad peroxidaseconjugate was added for one hour at room temperature. Determinations of actC3 were performed by colorimetric analysis of the peroxidase-mediated hydrolysis of $2 \mathrm{mM}$ 2,2'-azino-di-(3-ethyl benzthiazolinesulfonate) (ABTS, Boehringer Mannheim, Germany) in substrate buffer $(100 \mathrm{~m}$ sodium acetate, $50 \mathrm{~m}$ sodium phosphate, $\mathrm{pH} 4.2$ ) containing 2.5 mrvi H202. Absorbance was measured at $410 \mathrm{~nm}$ (reference wavelength $490 \mathrm{~nm}$ ) using a microplate photometer (ThermoMax; Molecular Devices, Menlo Park, CA, USA). ActC3 concentrations in the samples were calculated from calibration curves obtained by plotting the absorbance of purified protein $\mathrm{C} 3 \mathrm{~b}$ on a double log scale.

(2) CH50 ELIZA measures: we dispensed each serum sample, the reference calibrator and control and a not solubilising control in an Eppendorf tube, the tube vortexed and inverted few times to be sure that the solution is well mixed, and incubated 2 hours at $37 \mathrm{c}$, and centrifuged at 10000-13500 xg "RCF" for 15 minutes, we calculate the mean of the absorbencies (OD) of reference calibrator, control and of each sample, and the result expressed as $\mathrm{CH} 50$ values.

\section{Statistical analysis}

the collected data were computerized and analyzed SPSS 18.0 for windows (SPSS Inc., Chicago, IL, USA) and MedCalc 13 for windows (MedCalc Software bvba, Ostend, Belgium) Continuous variables were expressed as the mean \pm SD \& median (range), and the categorical variables were expressed as a number (percentage).

continuous variables were checked for normality by using Shapiro-Wilk test.

Mann Whitney U test was used to compare two groups non-normally distributed variables.

independent samples student t-test was used to compare two groups of normally distrbuted data.

categorical data were compared using Chisquare test (\$) or Fisher's exact test when appropriate.

The significance Level for all above mentioned statistical tests done: the threshold of significance is fixed at $5 \%$ level (P-value); p-value $<0.001$ indicates highly statistically significant (HS) results, p-value $<0.05$ indicates statistically significant (S), and p-value $\geq 0.05$ indicates statistically insignificant (NS). Validity of TVS was calculated using diagnostic performance depend on sample $2 \times 2$ contingency tables generation. Sensitivity, specificity, positive predictive value, negative predicitive value, accuracy were calculated.

\section{RESULTS}

Table (1): shows high significant increment in C3 level post isothermic dialysis than pre it.

\begin{tabular}{|c|c|c|l|l|}
\hline C3 & Pre Isothermic dialysis & Post isothermic dialysis & P value & Pignificance \\
\hline $\begin{array}{c}80-160 \\
\mathrm{Mg} / \mathrm{dl}\end{array}$ & $62.8 \pm 3$ & $115.65 \pm 4$ & 0.0001 & gh significant \\
\hline
\end{tabular}

Table (2): shows significant increment in $\mathrm{CH} 50$ level post isothermic dialysis than pre it.

\begin{tabular}{|c|c|c|c|c|}
\hline CH50 & $\begin{array}{c}\text { Pre isothermic } \\
\text { dialysis }\end{array}$ & $\begin{array}{c}\text { Post isothermic } \\
\text { dialysis }\end{array}$ & p value & Significance \\
\hline $101-300$ & $154.65 \pm 90$ & $199.35 \pm 85$ & 0.04 & Significant \\
\hline
\end{tabular}

Table (3): shows significant increment in C3 level post cold dialysis than pre it.

\begin{tabular}{|c|l|l|l|l|}
\hline $\mathbf{C 3}$ & Pre cold dialysis & Post cold dialysis & P value & Significance \\
\hline $80-160 \mathrm{mg} / \mathrm{dl}$ & $61.75 \pm 32$ & $148.705 \pm 55$ & 0.0001 & High significant \\
\hline
\end{tabular}

Table (4): shows significant increment in $\mathrm{CH} 50$ level post cold dialysis than pre it.

\begin{tabular}{|c|c|c|c|c|}
\hline CH50 & Pre cold dialysis & Post cold dialysis & P value & Significance \\
\hline $101-300$ & $168.95 \pm 81$ & $211.55 \pm 72$ & 0.0888 & Significant \\
\hline
\end{tabular}


ejhm.journals.ekb.eg

Table (5): comparison between cold and isothermic dialysis on C3 level as regard pre treatment and insignificant difference was shown.

\begin{tabular}{|c|c|c|c|l|}
\hline $\mathbf{C 3}$ & thermic dialysis & Cold dialysis & P value & Significance \\
\hline $80-160 \mathrm{mg} / \mathrm{dl}$ & $62.8 \pm 32$ & $61.75 \pm 32$ & 0.9186 & Insignificant \\
\hline
\end{tabular}

Table (6): comparison between cold and isothermic dialysis on CH50 level as regard pre treatment and insignificant difference was shown.

\begin{tabular}{|c|l|l|l|l|}
\hline CH50 & sothermic dialysis & re cold dialysis & P value & Significance \\
\hline $101-300$ & $154.65 \pm 90$ & $168.95 \pm 81$ & 0.6018 & Insignificant \\
\hline
\end{tabular}

Table (7): shows significant increment in level of $\mathrm{C} 3$ in post cold dialysis than post isothermic dialysis

\begin{tabular}{|c|l|l|l|l|}
\hline $\mathbf{C 3}$ & isothermic dialysis & st cold dialysis & P value & Significance \\
\hline $80-160 \mathrm{mg} / \mathrm{dl}$ & $115.65 \pm 34$ & $148.705 \pm 55$ & 0.0297 & Significant \\
\hline
\end{tabular}

Table (8): shows insignificant difference in level of CH50 in post cold dialysis than post isothermic dialysis

\begin{tabular}{|l|l|l|l|l|}
\hline CH50 & isothermic dialysis & st cold dialysis & P value & Significance \\
\hline $101-300$ & $199.35 \pm 85$ & $211.55 \pm 72$ & 0.6301 & Insignificant \\
\hline
\end{tabular}

\section{DISCUSSION}

hemodialysis (HD) is the routine renal replacement therapy for more than 300,000 patients in the United States who have reached end-stage renal disease. The goals of HD are straightforward and include restoring the bodies' intracellular and extracellular fluid environment and accomplishing solute balance by either removal from the blood into the dialysate or from the dialysate into the blood. Optimal care of the patient receiving long-term HD requires appropriate prescreption according to patientand device-dependent variables ${ }^{(6)}$.

the dialysate temperature is traditionally set to $37^{\circ}$ as a means of keeping stable the HD patient's body core temperature and maintain isothermia. In reality however, the "warm dialysis" does not maintain isothermia but induce a mild hyper-thermic effect which on average, rise the core temperature by approximately $0.5^{\circ}-0.7^{\circ}$ Celsius, In "cold" dialysis, the temperature of the dialysate is set at least one degree below the standard dialysate temperature, with the majority of the cold dialysis studies using dialysate temperatures between $35^{\circ}-36^{\circ} \mathrm{C}^{(7)}$.

the benefits of the cold dialysis in patients' health are based on the fact that lowering the body core temperature will improve systemic vascular resistance and therefore improve hemodynamic stability ${ }^{\left({ }^{(8)}\right.}$.

we included 20 patients in our study which their mean age 46.3 years, 11 males and 9 female, their mean BMI $82 \mathrm{~kg} / \mathrm{m}^{2}$ and the all patients hemodialysate via arterio-venous fistula.

this is the first study investigating the kinetics of $\mathrm{C} 3$ and $\mathrm{CH} 50$ of the complement pathway during hemodialysis. total level of $\mathrm{C} 3$ and $\mathrm{CH} 50$ at 60 min were significantly higher than those at $0 \mathrm{~min}$. hemodialysis is responsible for that increasment. our study results comes in agreement with Hirouki et al. ${ }^{(9)}$ who found that when he obtained serum samples from five patients during hemodialysis to investigate the kinetics of complement components, he found that the levels of $\mathrm{C} 3$ and $\mathrm{CH} 50$ complexes at $60 \mathrm{~min}$ were significantly increased when compared with those at $0 \mathrm{~min}$.

when we comparing the pre dialysis figure of both $\mathrm{C} 3$ and $\mathrm{CH} 50$, we noticed that both $\mathrm{C} 3$ and $\mathrm{CH} 50$ returned back to its pre dialysis values, which gave us the impression that activation of the complement system during the session is time limited. our study comes in agreement with Felix $\boldsymbol{e t}$ al. ${ }^{(\mathbf{1 0})}$ who studied generation of plasma C5-9 complex with $\mathrm{C} 3$ and C4 fragments in 29 patients during hemodialysis, he noticed that there were increase in $\mathrm{C} 4$ and $\mathrm{C} 3$ complex 15 minutes after initiation of hemodialysis procedure, they reach to their plateau after 120 minutes, $\mathrm{C} 4$ shown continual increase but $\mathrm{C} 3$ shown minimal decline.

our study also investigate cold hemodialysis is associated with more significant activation of complement system. That comes in agreement with Tushar et al. ${ }^{(11)}$ who measured the independent effect of temperature on complement activation, and more specifically, examine the relationship between clinical hypothermia temperatures $\left(31-33^{\circ} \mathrm{C}\right)$, and complement activation. he used Antibody-sensitized erythrocytes to assay complement activation at temperatures ranging from $0-41{ }^{\circ} \mathrm{C}$. Individual complement pathway components were assayed by ELISA, Western blot, and quantitative dot blot. Antibody-initiated complement activation resulting in eukaryotic cell lysis was increased by 2 -fold at $31^{\circ} \mathrm{C}$ compared with $37^{\circ} \mathrm{C}$. Antibody-initiated complement activation in human serum increased as temperature decreased from $37^{\circ} \mathrm{C}$ until dramatically decreasing at $13^{\circ} \mathrm{C}$. Quantitation of individual complement components showed significantly increased activation of $\mathrm{C} 4, \mathrm{C} 3$, and $\mathrm{C} 5$ at clinical hypothermia temperatures.

\section{CONCLUSION}

cold hemodialysis is associated with activation of complement system. 


\section{REFERENCES}

1. Cheung AK (1990): Biocompatibility of hemodialysis membranes. J. Am. Soc. Nephrol., 1: 150-161.

2. Eldehni MT, Odudu A, McIntyre CW (2015): Randomized clinical trial of dialysate cooling and effects on brain white matter. J Am Soc Nephrol., 26: 957-965.

3. Felix P, Faria B, Gaya da Costa $M$ et al. (2018): The Complement System in Dialysis: A Forgotten Story?. Frontiers in Immunology, 9:71.

4. Hiroyuki I, Isao O, Kisara O et al. (2012): An analysis of functional activity via the three complement pathways during hemodialysis sessions: A new insight into the association between the lectin pathway and C5 activation.Clinical Kidney Journal, 5(5): 401-404.

5. Ikizler AT and Schulman G (2005): Hemodialysis: Techniques and prescription. American Journal of Kidney Diseases, 46(5): 976-981

6. Kourtzelis I, Markiewski MM, Doumas M et al. (2010): Complement anaphylatoxin C5a contributes to hemodialysis -associated thrombosis. Blood, 116:631639.

7. Mustafa RA, Bdair F, Akl EA et al. (2016): Effect of Lowering the Dialysate Temperature in Chronic Hemodialysis: A Systematic Review and MetaAnalysis. Clin J Am Soc Nephrol., 11: 442-457.

8. Nilsson B, Mollnes KN, Lambris TE (2007): The role of complement in biomaterial-induced inflammation. Mol. Immunol., 44 (1): 82-94.

9. Robert AD, Edimara SR, Daniel R et al. (2012): Targeted complement inhibition as a promising strategy for preventing inflammatory complications in hemodialysis. Immunobiology, 217(2): 1097-1105.

10. Selby NM, McIntyre CW (2006): A systematic review of the clinical effects of reducing dialysate fluid temperature. Nephrol Dial Transplant., 21: 1883-1898.

11. Tushar SA, Mauriello CT, Hair PS et al. (2014): Clinical hypothermia temperatures increase complement activation and cell destruction via the classical pathway. Journal of Translational Medicine, 12(1):181. 